Draft Version May 31, 2021

Preprint typeset using $\mathrm{LAT}_{\mathrm{E}} \mathrm{X}$ style emulateapj v. 5/2/11

\title{
ORIGIN OF NE EMISSION LINE OF VERY LUMINOUS SOFT X-RAY TRANSIENT MAXI J0158-744
}

\author{
Yukari Ohtani $^{1,3}$, Mikio MoriI ${ }^{2}$ And Toshikazu Shigeyama ${ }^{3}$ \\ Draft version May 31, 2021
}

\begin{abstract}
We investigate the mechanism to reproduce notable spectral features at the ignition phase of nova explosion observed for a super-Eddington X-ray transient source MAXI J0158-744 in the Small Magellanic Cloud. These are a strong Ne IX emission line at $0.92 \mathrm{keV}$ with a large equivalent width of $0.32_{-0.11}^{+0.21} \mathrm{keV}$ and the absence of $\mathrm{Ne} \mathrm{X}$ line at $1.02 \mathrm{keV}$. In this paper, we calculate the radiative transfer using a Monte Carlo code, taking into account the line blanketing effect due to transitions of $\mathrm{N}, \mathrm{O}, \mathrm{Ne}, \mathrm{Mg}$ and $\mathrm{Al}$ ions in an accelerating wind emanating from a white dwarf with a structure based on a spherically symmetric stationary model. We found that the strong Ne IX line can be reproduced if the mass fraction of $\mathrm{Ne}$ is enhanced to $10^{-3}$ or more and that of $\mathrm{O}$ is reduced to $\sim 5 \times 10^{-9}$ or less and that the absence of other lines including $\mathrm{Ne} \mathrm{X}$ ions at higher energies can be also reproduced by the line blanketing effect. This enhancement of the Ne mass fraction indicates that the ejecta are enriched by Ne dredged up from the surface of the white dwarf composed of $\mathrm{O}, \mathrm{Ne}$, and $\mathrm{Mg}$ rather than $\mathrm{C}$ and $\mathrm{O}$, as already pointed out in the previous work. We argue that the CNO cycle driving this nova explosion converted most of $\mathrm{C}$ and $\mathrm{O}$ into $\mathrm{N}$ and thus reduced the $\mathrm{O}$ mass fraction.

Subject headings: X-rays: individual (MAXI J0158-744) - novae, cataclysmic variables - white dwarfs - radiative transfer - scattering - line formation
\end{abstract}

\section{INTRODUCTION}

MAXI (Monitor of All-sky X-ray Image; Matsuoka et al. 2009) discovered a luminous soft X-ray transient, MAXI J0158-744, in the direction of the east edge of the Small Magellanic Cloud (SMC) on November 11, 2011 (Kimura et al. 2011). The spectra of the initial X-ray flash are reproduced by blackbody models with the temperature of $\sim 300 \mathrm{eV}$ and the photospheric radius of $\sim 2,000 \mathrm{~km}$ (Morii et al. 2013). The Swift follow-up observations, which started from 0.44 days after the MAXI discovery, revealed that the $\mathrm{X}$-ray spectra, which are fitted by blackbody spectra at temperature $\sim 0.1 \mathrm{keV}$, were similar to those of novae in the super-soft source (SSS) phase (Li et al. 2012). The optical spectra taken by SAAO, ESO, and SMARTS telescopes indicated the characteristics of a Be star at the distance of the SMC (Li et al. 2012; Morii et al. 2013). Thus, MAXI J0158-744 was recognized as a nova accompanied by a Be star. The X-ray flash detected by MAXI GSC (Gas Slit Camera; Mihara et al. 2011; Sugizaki et al. 2011) and SSC (Solid-state Slit Camera; Tsunemi et al. 2010; Tomida et al. 2011) lasted for $\sim 1300 \mathrm{~s}$ at least. The maximum luminosity was $\sim 100$ times brighter than the Eddington limit of a solar mass object.

MAXI J0158-744 behaved differently from most novae known up to the present. While standard novae emit optical photons for at least several days before emitting soft X-ray photons during the SSS phase, the outburst of MAXI J0158-744 was first detected in the X-ray band, not in optical. Morii et al. (2013) reported that the un-

\footnotetext{
${ }^{1}$ Department of Astronomy, Graduate School of Science, University of Tokyo, Bunkyo-ku, Tokyo 113-0033, Japan

${ }^{2}$ MAXI team, Institute of Physical and Chemical Research (RIKEN), 2-1 Hirosawa, Wako, Saitama 351-0198, Japan.

${ }^{3}$ Research Center for the Early Universe, Graduate School of Science, University of Tokyo, Bunkyo-ku, Tokyo 113-0033, Japan
}

absorbed X-ray luminosity was about $3 \times 10^{39} \mathrm{erg} \mathrm{s}^{-1}$ at $t=8 \mathrm{~s}\left(=t_{8}\right)$ in the energy range of $0.7-7.0 \mathrm{keV}$, assuming the distance of $60 \mathrm{kpc}$ (Hereafter, the origin of time is referred to the MAXI trigger time.). The X-ray luminosity reached the peak of $L_{\mathrm{p}} \sim 2 \times 10^{40} \mathrm{erg} \mathrm{s}^{-1}$ at $t=220 \mathrm{~s}$ $\left(=t_{220}\right)$, and then decreased to $L_{1296} \sim 7 \times 10^{39} \mathrm{erg} \mathrm{s}^{-1}$ at $t=1296 \mathrm{~s}\left(=t_{1296}\right)$. Since these luminosities exceed the Eddington luminosity for a solar mass object by a factor of more than 10, a strong wind is expected to blow from the surface of the white dwarf (WD). This source is also characterized by a lack of the early optical phase and the very fast decay of the initial X-ray flash. The scan periods of the MAXI detectors constrain the duration $\Delta t$ of the event to be less than $1.10 \times 10^{4} \mathrm{~s}$. If the energy emitted in the X-ray band is supplied by hydrogen burning, we can estimate the mass of the exhausted hydrogen fuel as $M_{\mathrm{H}}=L_{\mathrm{p}} \Delta t / 0.007 c^{2} \sim 2 \times 10^{-8} M_{\odot}$, which is much smaller than those in usual novae $\left(\gtrsim 10^{-7} M_{\odot}\right.$; Fuiimoto 1982). Because of this tiny mass, the wind emanating from the WD becomes transparent soon after the thermonuclear runaway (TNR) outbreak. Especially, the most remarkable feature is the X-ray spectrum taken by MAXI/SSC at $t=t_{1296}$, which exhibits a strong Helike $\mathrm{Ne}$ (Ne IX) emission line at the energy of $0.92 \mathrm{keV}$ and no prominent line of $\mathrm{H}$-like $\mathrm{Ne}(\mathrm{Ne} \mathrm{X})$ at $1.02 \mathrm{keV}$ (Morii et al. 2013). We adopt the results of the analysis by Morii et al. (2013) using blackbody fits, defining $R_{1296}=2,290 \mathrm{~km}$ and $k_{\mathrm{B}} T_{1296}=0.33 \mathrm{keV}$ (where $k_{\mathrm{B}}$ is the Boltzmann constant) as fiducial values of the photospheric radius and temperature at $t=t_{1296}$.

Though Li et al. (2012) tried to explain the superEddington luminosity by a shock induced model, Morii et al. (2013) pointed out the difficulty in such a shock-induced model. The latter authors instead argued that the observed phenomenon corresponds to the fireball phase at the ignition of a nova explosion and that the luminosity is possibly explained by the convection on 
the surface of the WD, releasing a large amount of energy produced by the TNR during the first $100 \mathrm{~s}$. They inferred that the He-like Ne line can be explained by the optically thin region around the photosphere. In their work, optically thin emission component with a temperature below $0.3 \mathrm{keV}$ (Mekal component in Morii et al. 2013) and exceptionally large Ne abundance of 10 solar or more was necessary to explain the strong He-like $\mathrm{Ne}$ line and the lack of the $\mathrm{H}$-like $\mathrm{Ne}$ line. In this case, a large emission measure $\left(\sim 10^{63} \mathrm{~cm}^{-3}\right)$ is necessary and might be incompatible with the assumption of optically thin emission.

In this paper, we investigate the possibility that photons scattered by $\mathrm{N}, \mathrm{O}, \mathrm{Ne}, \mathrm{Mg}$, and $\mathrm{Al}$ ions in a supersonic wind would exclusively form a strong $\mathrm{K} \alpha$ line of He-like $\mathrm{Ne}$ and H-like N with P-Cygni profiles in the spectrum taken at $t=t_{1296}$ and eliminate other lines by line blanketing effects. The line blanketing occurs when the Doppler broadened line profile overlaps with that of another line at a higher energy or shorter wavelength, and weakens the latter line and strengthen the former line. A supersonic wind is expected to enhance this effect owing to large Doppler shifts of the line energies. We use a Monte Carlo method to calculate the photon transfer and try to reproduce the spectral features at $t=t_{1296}$.

In Section 2, we present our simplified stationary wind model including the velocity and density profiles and describe the procedure to determine the mass loss rate from observed quantities. The radiative processes involved in our Monte Carlo method are presented in Section 3 . In Section 4 we show the results and compare them with observations. We conclude this paper in Section 5

\section{MODEL OF ACCELERATING WIND}

To reproduce the spectrum at $t=t_{1296}$, we construct a model of nova wind by solving simplified equations for a spherically symmetric wind above the photosphere. Because of the super-Eddington luminosity of MAXI J0158-744, the gas pressure is significantly smaller than the radiation pressure. Therefore the equation of motion can be approximated as

$$
v \frac{d v}{d r}=\frac{\kappa L}{4 \pi r^{2} c}\left(1-\frac{L_{\mathrm{Edd}}}{L}\right),
$$

where $v$ is the velocity, $r$ the radial distance from the center, $\kappa$ the opacity, $L$ the luminosity, $c$ the speed of light. The Eddington luminosity $L_{\mathrm{Edd}}$ of a WD with the mass $M_{\mathrm{WD}}$ has been introduced as $L_{\mathrm{Edd}}=4 \pi c G M_{\mathrm{WD}} / \kappa$, where $G$ denotes the gravitational constant. Here we have assumed that the wind becomes stationary, because the time $t_{1296}$ is much longer than the dynamical timescale of this wind, that is $t_{\mathrm{d}} \sim R_{1296} / v=1-0.1 \mathrm{~s}$ for the typical wind velocity of novae $\left(v \sim 10^{3}-10^{4} \mathrm{~km}\right.$ $\mathrm{s}^{-1}$; Schwarz et al. 2011). The solution of Equation (1) is expressed as

$$
v=\sqrt{\frac{\kappa L}{2 \pi c R_{0}}\left(1-\frac{L_{\mathrm{Edd}}}{L}\right)\left(1-\frac{R_{0}}{r}\right)+v_{0}^{2}},
$$

where $R_{0}$ is the photospheric radius, which is equal to $R_{1296}$ at $t=t_{1296}$, and $v_{0}$ is the velocity at $r=R_{0}$. Note that $L_{\mathrm{Edd}} / L$ is of the order of 0.01 . The velocity at $r \rightarrow \infty$ approaches $v_{\max } \sim \sqrt{\kappa L / 2 \pi c R_{0}}$ if $v_{0}<<$ $v_{\max }$. Here we have assumed that the luminosity $L$ is kept to be constant at $L_{1296}$ above the photosphere. The initial velocity $v_{0}$ needs to be significantly smaller than the maximum velocity $v_{\max }$ for the line blanketing to work efficiently. In the following, we set $v_{0}=100 \mathrm{~km}$ $\mathrm{s}^{-1}$.

The mass loss rate is determined so that the optical depth of the solution with respect to the planck mean opacity be $2 / 3$. To do this, we need to calculate the ionization states in the wind. The mass loss rate in turn affects the ionization states in the wind through the ionization parameter for a photo-ionized plasma. Therefore we need to iterate this procedure to obtain an appropriate mass loss rate.

\subsection{Ionization States}

We use the XSTAR subroutines (Kallman \& Bautista 2001) to calculate ionization states in the wind irradiated by the blackbody radiation with the temperature of $T=T_{1296}$ and the photospheric radius of $R_{0}=R_{1296}$. The bolometric luminosity becomes $8.0 \times 10^{39} \mathrm{erg} \mathrm{s}^{-1}$. Though the actual wind solution has a radial density profile, we treat the wind as a gas sphere with a constant density that reproduces the same column depth for simplicity. This assumption overestimates the ionization parameters near the photosphere and underestimates them in the outer layer. In addition, we do not include the adiabatic cooling in the code, which may dominate the cooling process in the supersonic wind. Instead, we assume a gas temperature $(33 \mathrm{eV})$ significantly smaller than the photospheric temperature to use the XSTAR with minimum modifications. Thus we obtain the ionization states of relevant elements as functions of the column depth measured from the photosphere (Figure 1). Assigning the ionization states to positions in the wind with the same column depth, we obtain the ionization states in the wind. We leave a detailed and self-consistent treatment of ionization states in supersonic winds as a future work.

The mass fraction of the heavy elements except for $\mathrm{CNO}$ and Ne is assumed to be 0.1 solar value (see Table [1] the solar abundance is taken from Anders \& Grevesse $(1989)$ ) in the wind to mimic the SMC abundance (Carrera et al. 2008). The compositions of CNO elements should be modified by the CNO cycle operating beneath the photosphere. We treat the Ne mass fraction as a free parameter to reproduce the observed strong $\mathrm{Ne}$ line. We will discuss this issue in Section 4

\begin{tabular}{|c|c|}
\hline & mass fraction \\
\hline $\mathrm{N}$ & $1.09 \times 10^{-4}$ \\
\hline $\mathrm{O}$ & $1.02 \times 10^{-3}$ \\
\hline $\mathrm{Ne}$ & $1.85 \times 10^{-4}$ \\
\hline $\mathrm{Mg}$ & $6.93 \times 10^{-5}$ \\
\hline $\mathrm{Al}$ & $6.20 \times 10^{-6}$ \\
\hline
\end{tabular}

TABLE 1

REFERENCE VALUE FOR THE MASS FRACTION OF EACH ELEMENT, CORRESPONDING TO $10 \%$ OF THE SOLAR ABUNDANCE, A TYPICAL SMC ABUNDANCE (ANDERS \& GREVESSE 1989). 


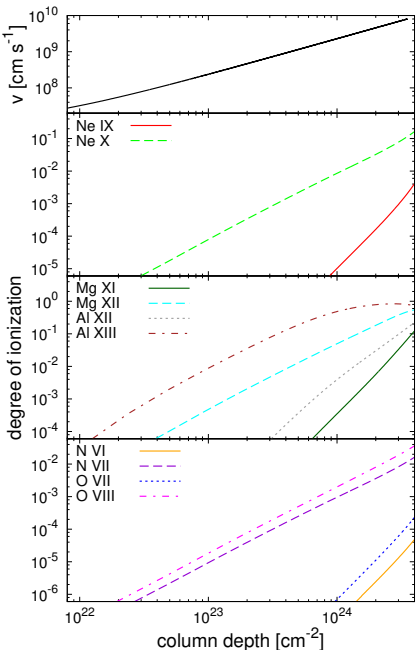

FIG. 1.- Distributions of expansion velocity (top panel) and degree of ionization (i.e., the ratio of ion density in each ionized state to the total density) for each element in the wind. The column depth is measured from the photosphere.

Resonance line scattering of photons in a spherically expanding wind would form P-Cygni profiles on the spectrum and may cause line blanketing effects depending on the velocity distribution in the wind. We calculate the transfer of photons in the expanding wind by a Monte Carlo simulation. Here we describe the boundary conditions and the treatment of radiative processes involved in the simulation.

\subsection{Transfer of Photons}

The calculation starts with photons isotropically emitted at the photosphere (at which the radius is $R_{1296}$ and the optical depth equals $2 / 3$ ). Their energy distribution is expressed by the Planck function with the temperature of $T_{0}$ in the rest frame of the wind, i.e.,

$$
B_{\mathrm{ph}, \epsilon^{\prime}}=\frac{2}{h^{3} c^{2}} \frac{\epsilon^{\prime 2}}{\exp \left(\epsilon^{\prime} / k_{\mathrm{B}} T_{0}\right)-1},
$$

where $\epsilon^{\prime}$ denotes the photon energy in the rest frame of the wind, $h$ is the Planck constant. We adopted $T_{0}=$ $T_{1296}$ suggested by the MAXI observation.

Seed photons with the energy of $\epsilon$ emitted from the photosphere can be absorbed by ions with the velocity $v$, only when the Doppler shifted energy $\epsilon^{\prime}$ of the photons in the rest frame of the ions match precisely the boundbound transition energy $\epsilon_{\text {lu }}$ of the ions, from the lower $(n=1)$ to upper $(n=\mathrm{u})$ state,

$$
\epsilon_{\mathrm{lu}}=\epsilon \gamma[1-(v / c) \cos \varphi] .
$$

Here $\epsilon$ is the photon energy in the fixed frame, $\gamma=$ $1 / \sqrt{1-(v / c)^{2}}$ is the Lorentz factor, $\varphi$ the angle between the momentum of a photon and the radial direction. Then the total cross section as a function of the photon energy $\epsilon^{\prime}$ in the ion rest frame is expressed as the Lorentz profile

$$
\sigma\left(\epsilon^{\prime}\right)=\frac{\pi r_{0} c \hbar^{2} A_{\mathrm{ul}}}{\left(\epsilon^{\prime}-\epsilon_{\mathrm{lu}}\right)^{2}+\left(\hbar A_{\mathrm{ul}} / 2\right)^{2}},
$$

where $\hbar$ denotes the Dirac constant and the Einstein $A$ coefficient is written by

$$
A_{\mathrm{ul}}=\frac{1}{g_{\mathrm{u}}} \frac{8 \pi^{2} r_{0} \epsilon_{\mathrm{lu}}^{2}}{c h^{2}} g_{\mathrm{l}} f_{\mathrm{lu}},
$$

where $g_{\mathrm{u}}$ and $g_{1}$ are the statistical weights of the upper and lower states, respectively, $r_{0}$ is the classical electron radius, and $f_{\text {lu }}$ the oscillator strength (Rybicki \& Lightman 1979). Since the spontaneous transition from the state $n=\mathrm{u}$ to the ground state is much faster than the reverse transition, we assume all ions are in the ground state in the transfer calculations. The fast spontaneous transition also defeats collisional deexcitations. Thus the whole process of absorption by exciting an ion followed by emission due to the reverse transition is recognized as scattering.

Using the cross section in Equation (5) we calculate the probability $P$ of scattering off an ion i expressed as $1-\exp \left[-\int_{l_{\mathrm{st}}}^{l_{\mathrm{ed}}} \sigma\left(\epsilon^{\prime}\right) n_{\mathrm{i}} d l\right]$, where $n_{\mathrm{i}}$ is the number density of the ions $\mathrm{i}$ and the integration is performed through the path of a photon from $l_{\mathrm{st}}$ to $l_{\mathrm{ed}}$. Since $d l$ is converted to the energy shift in the rest frame of the ion by $d r / \cos \varphi=(d r / d v)\left(d v / d \epsilon^{\prime}\right)\left(d \epsilon^{\prime} / \cos \varphi\right)$, where $d r / d v$ and $d v / d \epsilon^{\prime}$ is derived from Equation (10) and the Doppler effect equation (4), $P$ is replaced by

$$
P=1-\exp \left[-\int_{\epsilon_{\mathrm{st}}^{\prime}}^{\epsilon_{\mathrm{ed}}^{\prime}} \sigma\left(\epsilon^{\prime}\right) n_{\mathrm{i}} \frac{d r}{d v} \frac{d v}{d \epsilon^{\prime}} \frac{d \epsilon^{\prime}}{\cos \varphi}\right] .
$$

Note that $\epsilon_{\mathrm{st}}^{\prime}$ is larger than $\epsilon_{\mathrm{ed}}^{\prime}$, while $l_{\mathrm{st}}$ is smaller than $l_{\text {ed }}$. Here we approximate the integration of $\sigma\left(\epsilon^{\prime}\right) d \epsilon^{\prime}$ by $\sigma\left(\epsilon_{\text {lu }}\right) \Delta \epsilon^{\prime}$, where $\left|\Delta \epsilon^{\prime}\right|=\left(v_{\mathrm{t}} / c\right) \epsilon_{\text {lu }}$ denotes the line width due to thermal Doppler broadening (Sobolev 1960). Therefore $P$ can be written by

$$
P=1-\exp \left[-\sigma\left(\epsilon_{\mathrm{lu}}\right) n_{\mathrm{i}} \frac{d r}{d v} \frac{1-(v / c) \cos \varphi}{\cos ^{2} \varphi} v_{\mathrm{t}}\right],
$$

in the non-relativistic case.

Each ion follows the Maxwell-Boltzmann distribution in the rest frame of the wind,

$$
F_{\mathrm{i}} \propto p_{\mathrm{i}}^{2} \exp \left[-\frac{p_{\mathrm{i}}^{2}}{2 m_{\mathrm{i}} k_{\mathrm{B}} T_{\mathrm{i}}}\right],
$$

where $p_{\mathrm{i}}$ is the ion momentum, $m_{\mathrm{i}}$ is the ion mass, and $T_{\mathrm{i}}$ is the ion temperature.

The energy of the scattered photon is determined so that the energy distribution follows the Lorentz profile of Equation (5). Each photon repeats the scattering process described in this section until $t=t_{1296}$, unless it turns back into the photosphere.

In this study, we take into account 9 transitions from the ground states for He-like and H-like ions of $\mathrm{N}, \mathrm{O}, \mathrm{Ne}$, $\mathrm{Mg}$, and $\mathrm{Al}$, i.e., the upper states are $1 \mathrm{~s} 2 \mathrm{p}^{1} \mathrm{P}_{0}$ to $1 \mathrm{~s} 10 \mathrm{p}$. Table 2 lists the first two transitions of each ion included in our calculations.

\section{RESULTS}

First, we present results of our calculation taking only Ne lines into account and derive the required amount of $\mathrm{Ne}$ to produce a distinctive peak due to the $\mathrm{K} \alpha$ line of $\mathrm{Ne}$ IX. Figure 2 displays the dependence of the shape of the Ne line on the mass fraction, where $X_{\mathrm{Ne}}=1.85 \times 10^{-4}$ 


\begin{tabular}{|c|c|c|c|c|}
\hline & $\begin{array}{c}\text { ionization } \\
\text { state }\end{array}$ & $\begin{array}{l}\text { upper } \\
\text { state }\end{array}$ & $\begin{array}{c}\text { energy } \\
{[\mathrm{keV}]}\end{array}$ & $\begin{array}{l}\text { oscillator } \\
\text { strength }\end{array}$ \\
\hline \multirow{4}{*}{$\mathrm{O}$} & \multirow{2}{*}{ He-like } & $1 \mathrm{~s} 2 \mathrm{p}$ & 0.57 & 0.69 \\
\hline & & $1 \mathrm{~s} 3 \mathrm{p}$ & 0.67 & 0.15 \\
\hline & \multirow{2}{*}{ H-like } & $1 \mathrm{~s} 2 \mathrm{p}$ & 0.65 & 0.42 \\
\hline & & $1 \mathrm{~s} 3 \mathrm{p}$ & 0.77 & 0.08 \\
\hline \multirow{4}{*}{$\mathrm{Ne}$} & \multirow{2}{*}{ He-like } & $1 \mathrm{~s} 2 \mathrm{p}$ & 0.92 & 0.72 \\
\hline & & $1 \mathrm{~s} 3 \mathrm{p}$ & 1.07 & 0.15 \\
\hline & \multirow{2}{*}{ H-like } & $1 \mathrm{~s} 2 \mathrm{p}$ & 1.02 & 0.42 \\
\hline & & $1 \mathrm{~s} 3 \mathrm{p}$ & 1.21 & 0.08 \\
\hline \multirow{4}{*}{$\mathrm{Mg}$} & \multirow{2}{*}{ He-like } & $1 \mathrm{~s} 2 \mathrm{p}$ & 1.35 & 0.74 \\
\hline & & $1 \mathrm{~s} 3 \mathrm{p}$ & 1.58 & 0.15 \\
\hline & \multirow{2}{*}{ H-like } & $1 \mathrm{~s} 2 \mathrm{p}$ & 1.47 & 0.42 \\
\hline & & $1 \mathrm{~s} 3 \mathrm{p}$ & 1.74 & 0.08 \\
\hline \multirow{4}{*}{$\mathrm{Al}$} & \multirow{2}{*}{ He-like } & $1 \mathrm{~s} 2 \mathrm{p}$ & 1.60 & 0.75 \\
\hline & & $1 \mathrm{~s} 3 \mathrm{p}$ & 1.87 & 0.15 \\
\hline & \multirow{2}{*}{ H-like } & $1 \mathrm{~s} 2 \mathrm{p}$ & 1.72 & 0.42 \\
\hline & & $1 \mathrm{~s} 3 \mathrm{p}$ & 2.04 & 0.08 \\
\hline
\end{tabular}

TABLE 2

A LIST OF REPRESENTATIVE TRANSITIONS ADOPTED IN OUR Calculations. The values are fetched from the atomic line LIST VERSION 2.04 OF PETER VAN HOOF (SEE HTTP://WWW.PA.UKY.EDU/ PETER/ATOMIC/).

(green dash-dotted line), $1.0 \times 10^{-3}$ (orange dashed line), and $10^{-2}$ (red solid line). The cyan dotted line represents the blackbody continuum with the temperature of $T_{1296}$ emitted from the photosphere at the radius of $R_{1296}$. When the Ne mass fraction is less than $10^{-3}$, a broad Ne line is formed and the peak appears at an energy higher than $0.92 \mathrm{keV}$ (the transition energy of Ne IX K $\alpha$ in the rest frame), which is incompatible with the MAXI observation. This is because Ne IX K $\alpha$ line becomes optically thin with this small Ne mass fraction while Ne X $\mathrm{K} \alpha$ line is still optically thick. Instead, when Ne IX $\mathrm{K} \alpha$ line is optically thick due to the large Ne mass fraction, photons emitted by $\mathrm{Ne} \mathrm{X} \mathrm{K} \alpha$ line at the energy of 1.02 $\mathrm{keV}$ in the rest frame of the ions are scattered by $\mathrm{K} \alpha$ transitions of Ne IX ions because the energy of the photons is reduced by as much as $\sim 17 \%$ and can become exactly $0.92 \mathrm{keV}$ in the rest frame of some Ne IX ions in the surrounding matter receding at velocities of the order of $v_{\max } \sim 50,000 \mathrm{~km} \mathrm{~s}^{-1}$. This scattering off a Ne IX ion degrades the photon energies lower than $0.92 \mathrm{keV}$ in the rest frames of the other ions. As a consequence, the existence of $\mathrm{Ne} \mathrm{X}$ ions does not form an emission line at $1.02 \mathrm{keV}$ but enhances the $\mathrm{K} \alpha$ line of Ne IX ions. This is the so-called line blanketing effect. Therefore we assume $X_{\mathrm{Ne}}=1 \times 10^{-2}$ in the following calculations so that a distinctive Ne peak surely appears at $0.92 \mathrm{keV}$. The mass fraction of $\mathrm{Ne}$ ions in the lower ionization stages is too low to affect the spectrum.

Next, we investigate effects of other abundant ions, i.e., Mg XI, XII, and Al XII, XIII. These ions have transitions with energies higher than the energy of the Ne X $\mathrm{K} \alpha$ line $1.02 \mathrm{keV}$. The green dash-dotted line in Figure 3 designates the result. The emission lines associated with transitions of $\mathrm{Mg}$ and $\mathrm{Al}$ ions look rather weak, and it is remarkable that the existence of these elements enhances the Ne line due to line blanketing (This enhancement is verified by comparison with the red solid line in Figure 2). Figure 4 illustrates a schematic view of the typical motion of scattered photons. The upper illustration is a schematic image of photons traveling in the wind

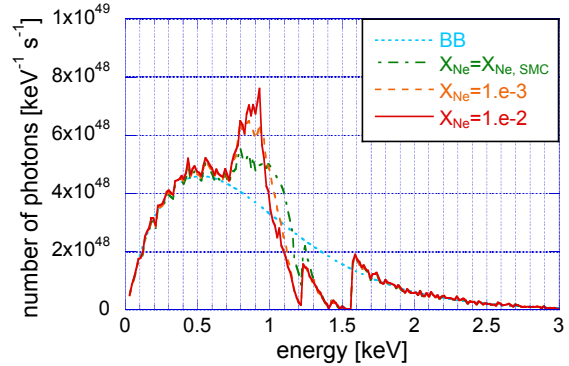

FIG. 2.- Spectra as results of Monte Carlo simulations taking into account only scattering by $\mathrm{Ne}$ with different mass fractions. The cyan dotted line is the incident irradiating emission represented by a blackbody spectrum at temperature $T=T_{1296}$. The other three lines represent the spectra with $X_{\mathrm{Ne}}=1.85 \times 10^{-4}$ (the same as the SMC abundance; green dash-dotted line), $X_{\mathrm{Ne}}=$ $1 \times 10^{-3}$ (the orange dashed line), and $X_{\mathrm{Ne}}=10^{-2}$ (the red solid line).

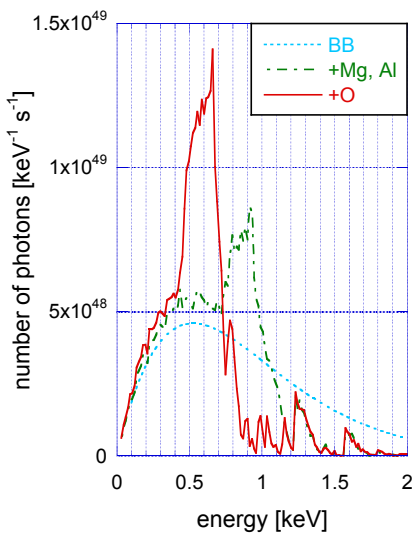

FIG. 3.- Comparison of spectra taking account of scattering off other ions as well as Ne. The cyan dotted line is the incident irradiating emission represented by a blackbody spectrum at temperature $T=T_{1296}$. The green dash-dotted line includes $\mathrm{Mg}$ XI, XII \& Al XII, XIII and the red solid line represents the spectrum including O VII and VIII in addition to these ions.

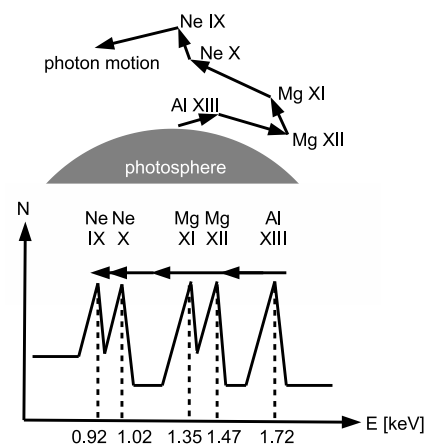

FIG. 4.- A schematic image of a representative photon track, which is scattered several times by different species of ions (upper illustration), and the spectral deformation (line blanketing effects; lower illustration).

and scattered by different species of ions in order of decreasing transition energy such as $\mathrm{Al}$ XIII $\rightarrow \mathrm{Mg}$ XII $\rightarrow \mathrm{Mg}$ $\mathrm{XI} \rightarrow \mathrm{Ne} \mathrm{X} \rightarrow \mathrm{Ne}$ IX. The lower illustration shows how this series of scattering changes the spectrum. Because the photon reduces its energy, the emission lines at energies higher than $0.92 \mathrm{keV}$ become weak. Figure 5 displays an example of decreasing energy of a photon with time, extracted from our calculation. The energy of a sample 


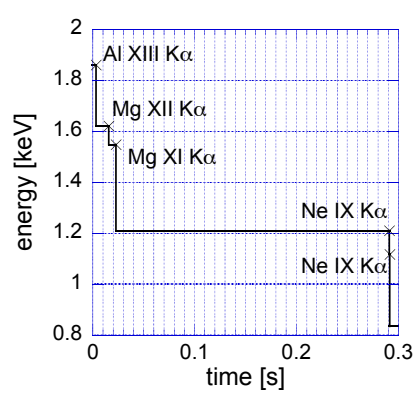

FIG. 5.- An example of photon energy shifts. The initial photon energy of $1.86 \mathrm{keV}$ decreases down to $0.83 \mathrm{keV}$ after scattering sequentially by several ions with transition energies in decreasing order, i.e., $\mathrm{Al}$ XIII $\mathrm{K} \alpha, \mathrm{Mg}$ XII $\mathrm{K} \alpha, \mathrm{Mg}$ XI $\mathrm{K} \alpha$, and $\mathrm{Ne}$ IX $\mathrm{K} \alpha$.

photon gradually changes from $1.86 \mathrm{keV}$ to $1.62,1.55$, $1.21,1.12$ and $0.83 \mathrm{keV}$ due to the scattering off Al XIII, Mg XII, Mg XI, Ne IX and Ne IX ions, respectively.

\subsection{Influence of Oxygen Mass Fraction on Ne line}

In contrast to the enhancement of the Ne line by the existence of $\mathrm{Mg}$ and $\mathrm{Al}$ ions, the inclusion of $\mathrm{O}$ VII and VIII ions with the mass fraction of 0.1 solar, which have transitions with the energies lower than $0.92 \mathrm{keV}$, significantly weakens the $\mathrm{K} \alpha$ line of Ne IX and produces a very strong emission line at $\sim 0.7 \mathrm{keV}$ as shown by the red solid line in Figure 3 .

To reproduce the observed spectrum, the reduction of the oxygen mass fraction is necessary. As a next step, we calculate spectra with reduced oxygen mass fractions and the results are shown in Figure 6. Here, the influence on the Ne line profile becomes small when the $\mathrm{O}$ mass fraction is between $X_{\mathrm{O}}=5 \times 10^{-8}$ (green dotted line) and $5 \times 10^{-9}$ (red solid line). If $X_{\mathrm{O}}=5 \times 10^{-8}$ then the line at $0.92 \mathrm{keV}$ disappears and the $\mathrm{K} \alpha$ line of $\mathrm{O}$ VIII alternatively becomes prominent. So, we refer to the model with $X_{\mathrm{O}}=5 \times 10^{-9}$ as the fiducial model. The fiducial model has the mass loss rate of $\approx 9.1 \times 10^{19} \mathrm{~g}$ $\mathrm{s}^{-1}$.

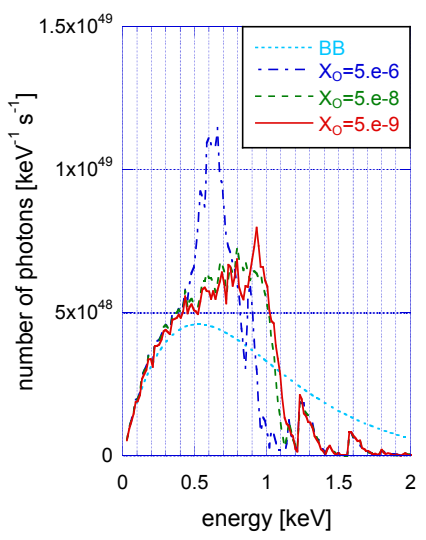

FIG. 6. - Influence of oxygen mass fraction on spectra, i.e., $X_{\mathrm{O}}=5 \times 10^{-6}$ (blue dash-dotted line), $5 \times 10^{-8}$ (green dashed line), and $5 \times 10^{-9}$ (red solid line). The cyan dotted line is the incident irradiating emission represented by a blackbody spectrum at temperature $T=T_{1296}$

\subsection{Oxygen Depletion in the Wind}

The reduction of the $\mathrm{O}$ mass fraction required to reproduce the observed spectrum can be realized by the
CNO cycle. To demonstrate this, we perform a nuclear reaction network calculation for a gas with a fixed temperature $\left(3 \times 10^{8} \mathrm{~K}\right)$ and density $\left(100 \mathrm{~g} \mathrm{~cm}^{-3}\right)$ using the code developed by one of the authors (Shigevama et al. 2010). The initial composition of heavy elements is 0.1 solar with the enhanced $\mathrm{O}$ and $\mathrm{Ne}$ mass fractions. The mass fraction of $\mathrm{Ne}$ is $X_{\mathrm{Ne}}=0.01$. The corresponding mass fraction of $\mathrm{O}$ becomes 0.4 solar $\left(X_{\mathrm{O}}=4.1 \times 10^{-3}\right)$ if we estimate from the surface composition of an ONe WD model with the mass of $1.37 M_{\odot}$ (Garcia-Berro \& Iben 1994). According to this modeling, the mass fraction of $\mathrm{O}$ on the surface of the core decreases with increasing stellar mass. Thus a WD with the mass closer to the Chandrasekhar limit leads to a smaller $X_{\mathrm{O}}$. Figure 7 shows the resultant temporal evolution of the composition of elements involved in the CNO cycle. Most of the initial CNO elements are converted to unstable isotopes ${ }^{15} \mathrm{O}$ and ${ }^{14} \mathrm{O}$ in $\sim 600 \mathrm{~s}$. Since the half life of ${ }^{15} \mathrm{O}\left({ }^{14} \mathrm{O}\right)$ is $122 \mathrm{~s}(71 \mathrm{~s})$, the required reduction of the $\mathrm{O}$ mass fraction suggests that the CNO cycle continued only for $\sim 10^{3} \mathrm{~s}$ and then the synthesized ${ }^{15} \mathrm{O}\left({ }^{14} \mathrm{O}\right)$ decayed to ${ }^{15} \mathrm{~N}\left({ }^{14} \mathrm{~N}\right)$ for the subsequent $\sim 2400 \mathrm{~s}$ or more.

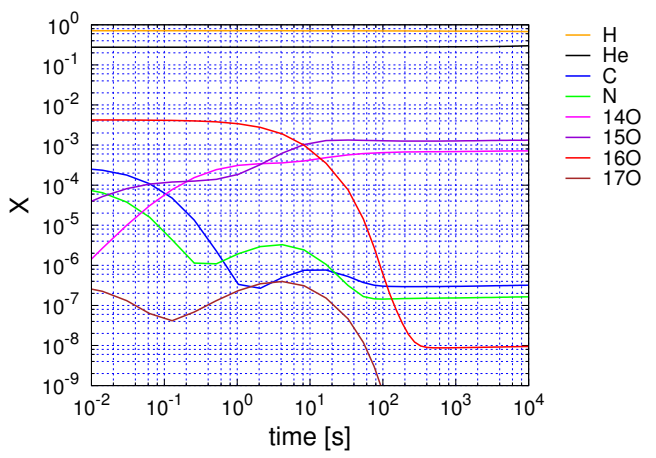

FIG. 7.- The evolution of the mass fraction of CNO elements. The CNO cycle reaction starts at $t=0$. Most of ${ }^{16} \mathrm{O}$ disappears in $\sim 600 \mathrm{~s}$ (from $X_{\mathrm{O}}=1 \times 10^{-3}$ to $4 \times 10^{-9}$ ), and replaced by ${ }^{15} \mathrm{O}$ and ${ }^{14} \mathrm{O} .{ }^{15} \mathrm{O}\left({ }^{14} \mathrm{O}\right)$ would decay into ${ }^{15} \mathrm{~N}\left({ }^{14} \mathrm{~N}\right)$ with the half-life time of $122 \mathrm{~s}(71 \mathrm{~s})$ after the end of the CNO cycle.

In other words, we need an interval of more than $\sim 2400$ $\mathrm{s}$ after the termination of the $\mathrm{CNO}$ cycle to eliminate $\mathrm{O}$ in the wind and to form a strong Ne line in the spectrum at $t=t_{1296}$. Then the CNO cycle must have stopped before $t \sim t_{1296}-2400 \mathrm{~s}=-1100 \mathrm{~s}$. No detection of the event by MAXI at $t=-5530 \mathrm{~s}\left(=t_{-5530}\right)$ further constrains the duration of the CNO cycle $\left(\Delta t_{\mathrm{CNO}}\right)$, that is $\Delta t_{\mathrm{CNO}}<-1100 \mathrm{~s}-t_{-5530}=4430 \mathrm{~s}$.

The resultant $\mathrm{O}$ mass fraction is two times higher than the required value derived in the previous section and the spectrum with this higher $\mathrm{O}$ mass fraction has the peak of the Ne IX line at a slightly higher energy $(0.95 \mathrm{keV})$ and marginally consistent with the observation. This might indicate that the WD is more massive than 1.37 $M_{\odot}$ and has a mass closer to the Chandrasekhar limit.

\subsection{Nitrogen}

Before finally coming to the conclusions, it is indispensable to examine whether $\mathrm{N}$ ions increased through the $\mathrm{O}$ decay argued in the former section is compatible with the observed Ne line or not. We calculate a spectrum including N VI and VII, assuming the same mass fraction 
of $\mathrm{N}$ as the initial $X_{\mathrm{O}}\left(=X_{\mathrm{O}, \mathrm{SMC}}=1 \times 10^{-3}\right)$. A list of mass fractions of elements we adopt in this calculation is shown in Table 3 .

The result is shown by the red solid line in Figure 8. In comparison with the calculation of "no N" (green dashdotted line), the existence of $\mathrm{N}$ sharpens the shape of the $\mathrm{Ne}$ line. It is again caused by line blanketing due to N VI and VII ions. In this calculation, most photons forming the lower energy part of the Ne line $(\lesssim 0.87$ $\mathrm{keV}$ ) are scattered by $\mathrm{N}$ ions and converted to $\mathrm{N}$ line at lower energies. Here, the half width at $1 / \mathrm{e}$ times the $\mathrm{Ne}$ peak above the blackbody heights is $0.080 \mathrm{keV}$, and the equivalent width is $0.18 \mathrm{keV}$, which are compatible with the observations (Morii et al. 2013).

A strong $\mathrm{N}$ line appears at $\sim 0.5 \mathrm{keV}$ close to the lower limit of the energy range covered by the MAXI/SSC. This must be compatible with the observation because the sensitivity at $0.5 \mathrm{keV}$ is a factor of about 3 weaker than that at $1 \mathrm{keV}$.

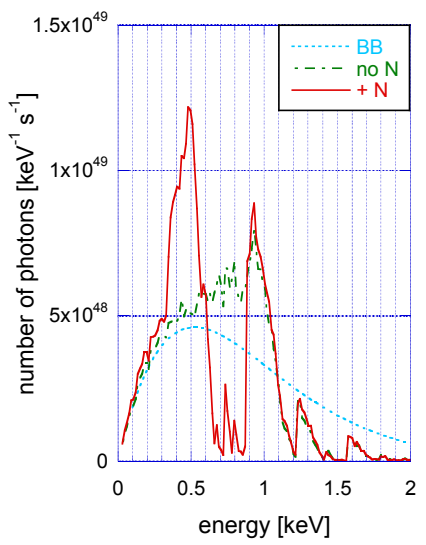

FIG. 8.- Comparison of spectra with (red solid line) and without (green dash-dotted line) N VI and VII transitions (The cyan dotted line is the incident irradiating emission represented by a blackbody spectrum at temperature $T=T_{1296}$.). These transitions scatter photons with the energy less than $0.87 \mathrm{keV}$, which should be recognized as a part of a broaden Ne line in the latter spectrum. The half width and the equivalent width of the Ne line (red solid line) are $0.080 \mathrm{keV}$ and $0.18 \mathrm{keV}$, respectively.

\begin{tabular}{|c|c|}
\hline & mass fraction \\
\hline $\mathrm{N}$ & $1.02 \times 10^{-3}$ \\
\hline $\mathrm{O}$ & $5.0 \times 10^{-9}$ \\
\hline $\mathrm{Ne}$ & $1.0 \times 10^{-2}$ \\
\hline $\mathrm{Mg}$ & $6.93 \times 10^{-5}$ \\
\hline $\mathrm{Al}$ & $6.20 \times 10^{-6}$ \\
\hline
\end{tabular}

TABLE 3

MASS FRACTION OF EACH ELEMENT ASSUMED IN THE FIDUCIAL MODEL. SEE TEXT FOR DETAILS.

\subsection{Influence of the Luminosity on Spectrum}

Because a luminosity derived from a blackbody fit to an observed spectrum has a large uncertainty in general (Krautter et al. 1996), we investigate how the luminosity affects the line blanketing. To do this, we decrease the value of luminosity in Equation (2) from that used in the former section to calculate ion scattering in the wind having lower velocities, while keeping the other parameter values such as the photospheric radius and the temperature unchanged as in Section 4.3. Figure9 shows the results. For $L=4 \times 10^{39} \mathrm{erg} \mathrm{s}^{-1}$, a prominent peak appears at $\mathrm{Ne} \mathrm{X} \mathrm{K} \alpha$ energy (the green dash-dotted line). For $L=2 \times 10^{39} \mathrm{erg} \mathrm{s}^{-1}$, the peak shifts to a lower energy. Therefore a luminosity brighter than $4 \times 10^{39} \mathrm{erg} \mathrm{s}^{-1}$ is required to enhance Ne IX K $\alpha$ line by line blanketing.

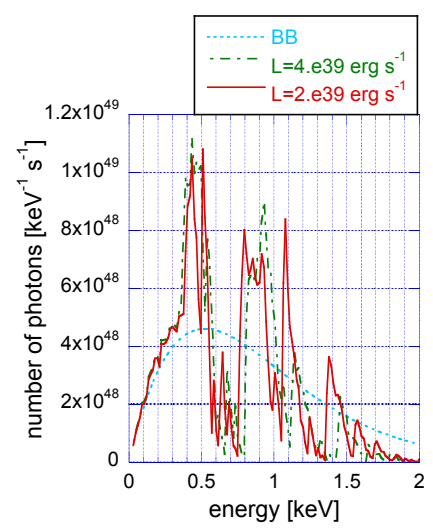

FIG. 9. - Spectra calculated for two values of the luminosity in Equation (2) $\left(4 \times 10^{39} \mathrm{erg} \mathrm{s}^{-1}\right.$ for the green dash-dotted line and $2 \times 10^{39} \mathrm{erg} \mathrm{s}^{-1}$ for the red solid line. The cyan dotted line is the blackbody continuum for $T=T_{1296}$.).

\section{CONCLUSIONS AND DISCUSSIONS}

We have examined whether resonance scattering off $\mathrm{N}$, $\mathrm{O}, \mathrm{Ne}, \mathrm{Mg}$, and $\mathrm{Al}$ ions in an accelerating wind reproduces a strong Ne IX K $\alpha$ emission line and lack of the $\mathrm{Ne} \mathrm{X} \mathrm{K} \alpha$ line in the observed X-ray spectrum of MAXI J0158-744, by performing Monte Carlo calculations. We have constructed a simplified stationary model for a given luminosity with the ion distribution calculated using the $\mathrm{XSTAR}$ subroutines. The mass loss rate is determined to reproduce the observed photospheric radius.

As a result of a series of the calculations presented here, the observed spectral features can be reproduced by emission from the expanding wind irradiated by a superEddington soft X-ray source. It is found that the Ne mass fraction needs to be enhanced by a factor of several tens of times compared with the SMC abundance $\left(X_{\mathrm{Ne}} \sim 1 \times\right.$ $10^{-2}$ ) to reproduce the observed Ne line at $0.92 \mathrm{keV}$. This amount of Ne could be supplied by matter dredged up from the surface of a WD composed of ONe. It should be noted that the ionization states estimated by ignoring the advection in the wind might overestimates the ionization degree and thus the necessary mass fraction of Ne. We also find that the inclusion of $\mathrm{Ne} \mathrm{X}$ ions at energies higher than $0.92 \mathrm{keV}$ does not induce the Ne X K $\alpha$ line due to the line blanketing. In the same way, inclusion of heavier elements like $\mathrm{Mg}$ and $\mathrm{Al}$ supply more photons to the $\mathrm{Ne}$ IX $\mathrm{K} \alpha$ line and strengthen it. Meanwhile, even a small amount of $\mathrm{O}$ weakens the $\mathrm{Ne}$ emission line for the same reason. It is necessary to decrease the mass fraction of $\mathrm{O}$ under $5 \times 10^{-9}$ for removing the influence on the Ne line completely. We have shown that the CNO cycle beneath the photosphere can reduce the amount of $\mathrm{O}$. To reduce its mass fraction under $5 \times 10^{-9}$, the duration of the $\mathrm{CNO}$ cycle is constrained to be shorter than $4430 \mathrm{~s}$. 
The inferred hydrogen mass burned out by the CNO cycle is $\approx 10^{-8} M_{\odot}$. This is far smaller than the minimum accretion mass that enables ignition in hydrostatic equilibrium, $5 \times 10^{-7} M_{\odot}$ (Fuijoto 1982). Taking this into account, hydrogen should be accreted dynamically onto the surface of the WD and the subsequently generated shock ignites hydrogen leading to TNR. Such a dynamical behavior may be responsible for the dredge up of Ne ions, which is needed to explain the observed Ne line. The mass of such a WD must be very close to the Chandrasekhar limit, which was also indicated from the small photospheric radii deduced from observations (Morii et al. 2013).

TS is grateful to Tatehiro Mihara for giving us an opportunity to think about modeling this very intriguing source and thanks Izumi Hachisu for useful comments.

\section{REFERENCES}

Anders, E., \& Grevesse, N. 1989, Geochim. Cosmochim. Acta, 53, 197

Carrera, R., Gallart, C., Aparicio, A., et al. 2008, AJ, 136, 1039

Fujimoto, M. Y. 1982, ApJ, 257, 767

Garcia-Berro, E., \& Iben, I. 1994, ApJ, 434, 306

Kallman, T., \& Bautista, M. 2001, ApJS, 133, 221

Kimura, M., Tomida, H., Sootome, T., et al. 2011, The Astronomer's Telegram, 3756, 1

Krautter, J., Oegelman, H., Starrfield, S., Wichmann, R., \& Pfeffermann, E. 1996, ApJ, 456, 788

Li, K. L., Kong, A. K. H., Charles, P. A., et al. 2012, ApJ, 761, 99

Matsuoka, M., Kawasaki, K., Ueno, S., et al. 2009, PASJ, 61, 999

Mihara, T., Nakajima, M., Sugizaki, M., et al. 2011, PASJ, 63, 623

Morii, M., Tomida, H., Kimura, M., et al. 2013, ApJ, 779, 118

Rybicki, G. B., \& Lightman, A. P. 1979, Radiative processes in astrophysics
Schwarz, G. J., Ness, J.-U., Osborne, J. P., et al. 2011, ApJS, 197, 31

Shigeyama, T., Nakamura, K., Tsujimoto, T., \& Moriya, T. 2010, in American Institute of Physics Conference Series, Vol. 1279, American Institute of Physics Conference Series, ed. N. Kawai \& S. Nagataki, 415-417

Sobolev, V. 1960, Moving envelopes of stars, Harvard books on astronomy

Sugizaki, M., Mihara, T., Serino, M., et al. 2011, PASJ, 63, 635

Tomida, H., Tsunemi, H., Kimura, M., et al. 2011, PASJ, 63, 397

Tsunemi, H., Tomida, H., Katayama, H., et al. 2010, PASJ, 62, 1371 